\title{
The impact of mycophenolate mofetil on androgen receptor activity in prostate cancer cells 22Rv1
}

Ondřei Ženata, Zdeněk Dvořák and Radim Vrzal

Email: zenataOndrej@seznam.cz

Department of Cell Biology and Genetics, Faculty of Science, Palacky University, Slechtitelu 27, 78371 Olomouc, Czech Republic

\section{Introduction:}

Mycophenolate mofetil (MYC) (Figure 1) is widely used immunosuppressive drug in the prevention of organ rejection after heart or kidney transplantation. It's primary target is the inhibition of inosine monophosphate dehydrogenase (IMPDH), enzyme which is essential in synthesis of the guanosine nucleotides. After oral administration, MYC is rapidly hydrolyzeded to mycophenolic acid (MPA) with high affinity to IMPDH II isoform, which is presented in primary lymphocytes.

Recently, our research group find that MYC is strong activator of aryl hydrocarbon receptor (AhR), which is involved cell differentiation, apoptosis and immunity. On the another hand, it acted like an antagonist in glucocorticoid receptor (GR) signalization since it abolished the effects of GR agonist dexamethasone (Vrzal et al., 2015).

Recent research show that some kinds of cancer are highly sensitive to MYC treatment (Dun et al., 2013). Treatment by AVN944, IMPDH II inhibitor, had antiproliferative effects and induced cell death in 4 cancer cell lines (Floryk et Thompson, 2008). Thus, similar effects may be expected for MYC. To that purpose, we decide to investigate the effect of MYC on the activity of androgen receptor (AR) in androgen-independend cell line (22Rv1) compared to androgen-dependent line (LNCap).

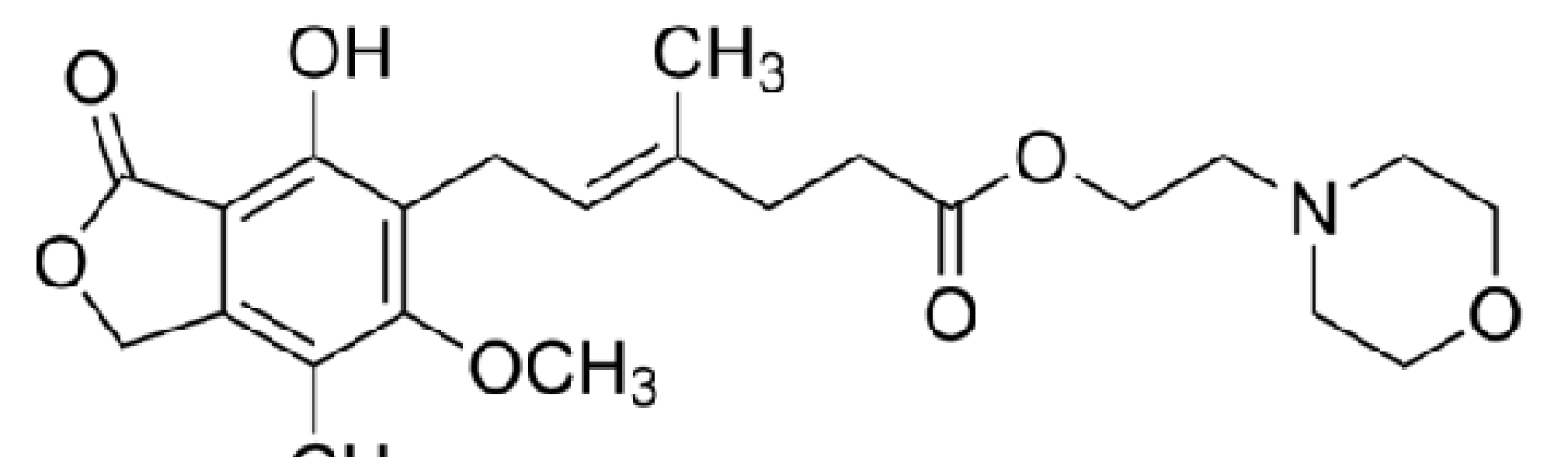

$\mathrm{CH}_{3}$

Figure 1: The structure of mycophenolate mofetil.

\section{Androgen receptor is activated by mycophenolate mofetil:}

As a first step, we had to determine non-toxic concentrations of MYC. We used the range correspondent with plasma concentrations (Pescovitz et al., 2000) with maximum 20 $\mu \mathrm{g} / \mathrm{ml}$ (approx. 62 $\mu \mathrm{M}$ ). In all used concentrations, there was no decrease by more than $10 \%$ in AIZ-AR cell line (Figure 2).

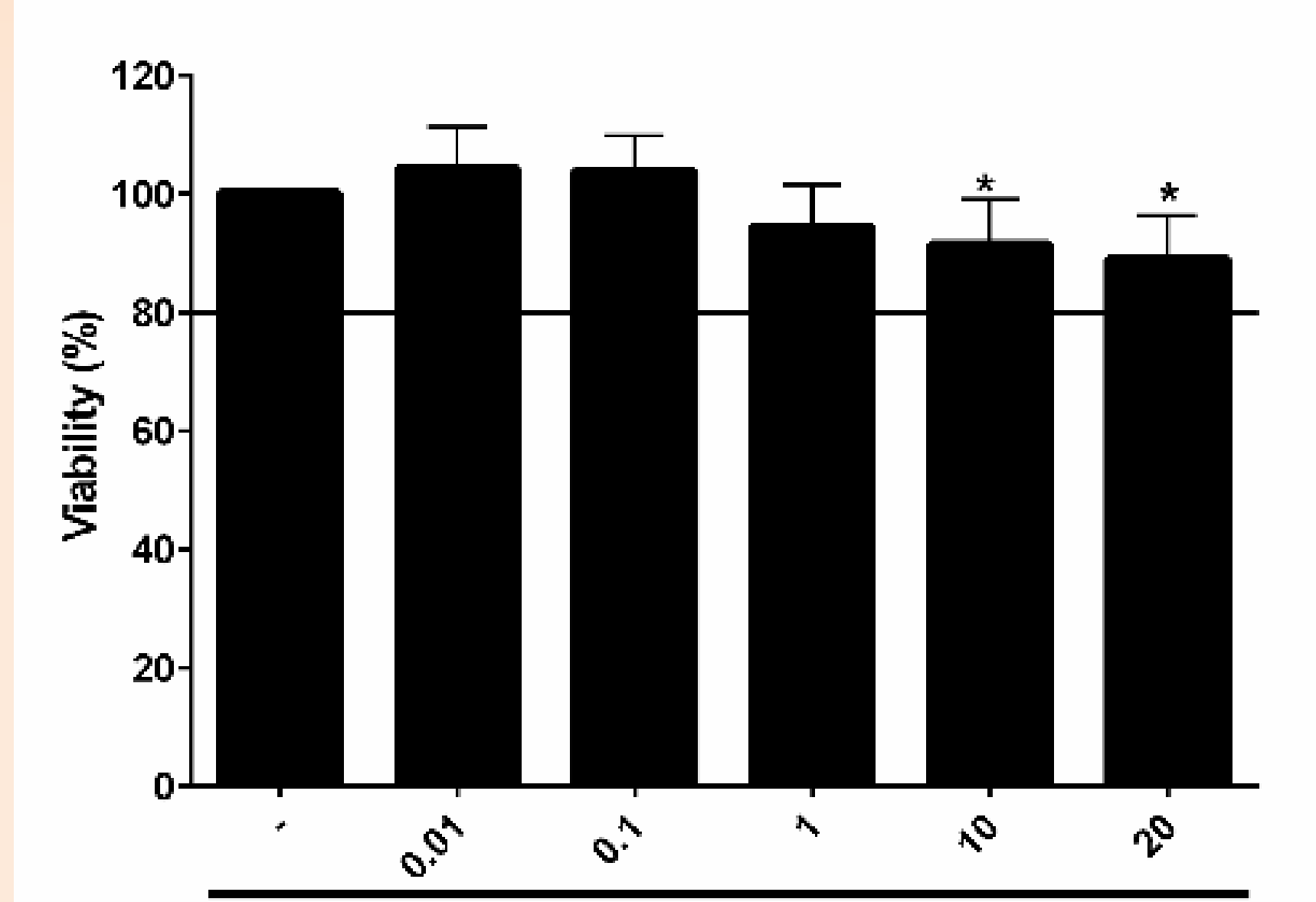

$\operatorname{MrC}(\mu \mathrm{g} / \mathrm{m})$

Figure 2: AIZ-AR cells were incubated with different concentrations of MYC $(0,01-20 \mu \mathrm{g} / \mathrm{ml})$ and/or DMSO $(0,1 \% \mathrm{v} / \mathrm{v})$ as a vehicle. After $24 \mathrm{~h}, \mathrm{MTT}$ assay was performed. Only two highest concentrations (10 and 20 $\mu \mathrm{g} / \mathrm{ml}$ ) significantly decreased the viability, but not over $20 \%{ }^{*}$ - value is significantly different from untreated cells (DMSO; $\mathrm{p}<0.05$ )

As a second step, we used recently developed AR-responsive cell line AIZ-AR, which has been derived from 22Rv1 cells transfected with a construct containing three copies of androgen response regions (ARRs) and one copy of androgen response element, respectively (Bartonkova et al., 2015) to determine if MYC can activate AR.

In the agonist setting, there was significant induction of luciferase activity $(1,4-1,7$ fold) for three highest concentrations $(1,10$ and $20 \mu \mathrm{g} / \mathrm{ml}$ ), see in Figure 3A. Dihydrotestosterone (DHT) was used as a positive control of functionality of the system (18-fold). In the Antagonist setting, we observed significant effect only for two lowest concentrations of MYC, which increased luciferase activity about 13\% above DHT alone (Figure 3B).

A
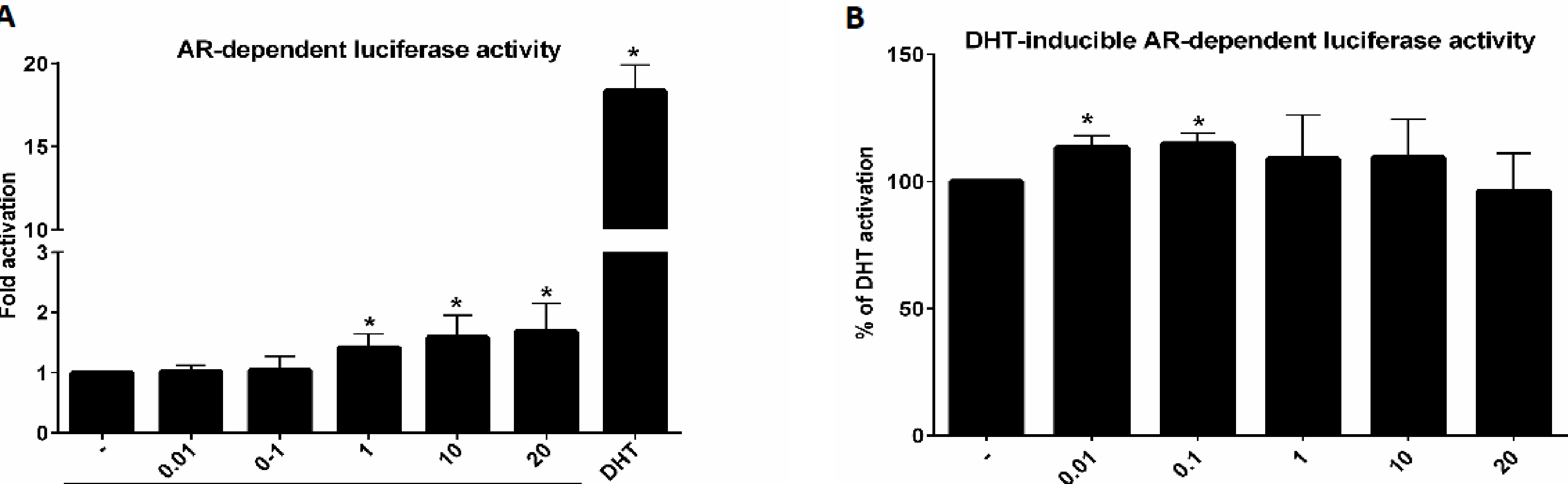

Figure 3: (A) AIZ-AR cells were incubated with different concentrations of Mycophenolate mofetil (MYC), DHT or DMSO $(0,1 \% \mathrm{v} / \mathrm{v})$ for $24 \mathrm{~h}$. The data are the mean \pm SD from five independent experiments and are expressed as fold activation of AR over DMSO-treated cells. (B) AIZ-AR cells were incubated with different concentrations of Mycophenolate mofetil (MYC) with DHT presence and DHT as positive control for $24 \mathrm{~h}$. The data are the mean \pm SD from seven independent experiments and are expressed as $\%$ of positive control $(\mathrm{DHT})$ * $^{*}$ - value is significantly different from untreated cells (DMSO; $\mathrm{p}<0.05$ ) (A) or DHT-treated cells (B).

\section{References:}

1. Vrzal R. et al., (2015): The effects of drugs with immunosuppressive or immunomodulatory activities on xenobiotics-metabolizing enzymes expression in primary human hepatocytes. Toxicol In Vitro 29:1088-99

Dun B. et al., (2013): Delineation of biological and molecular mechanisms underlying the diverse anticance activities of mycophenolic acid. Int J Clin Exp Pathol 6:2880-6

Floryk et Thompson (2008): Antiproliferative effects of AVN944, a novel inosine 5-monophosphate dehydrogenase inhibitor, in prostate cancer cells. Int J Cancer 123:2294-302

Pescovitz et al., (2000): Intravenous mycophenolate mofetil: safety, tolerability, and pharmacokinetics. Cl Transplant 14:179-88

Bartonkova et al., (2015): Novel stably transfected human reporter cell line

human androgen receptor transcriptional activity. PLOS One 10:e0121316
3. Mycophenolate mofetil modulates KLK3 expression and proliferation of prostate cells: One of the most known target genes of AR is KLK3 (kallikrein related peptidase 3), also known as PSA (prostate specific antigen). We observed effect of MYC on KLK3 mRNA in 22Rv1 (which the AIZ-AR cell line was derived from) and then on LNCap cell line. In 22Rv1, positive control significantly induced KLK3 mRNA about 2-fold, while MYC induced significantly about $60 \%$ strength od DHT for three highest concentrations (Figure 4A). Higher induction was observed in LNCap (approx. 15-fold) for positive control DHT, but surprisingly the highest MYC induction was only 1,5-fold for one concentration (about 3,5\% of DHT effect; Figure 4B).
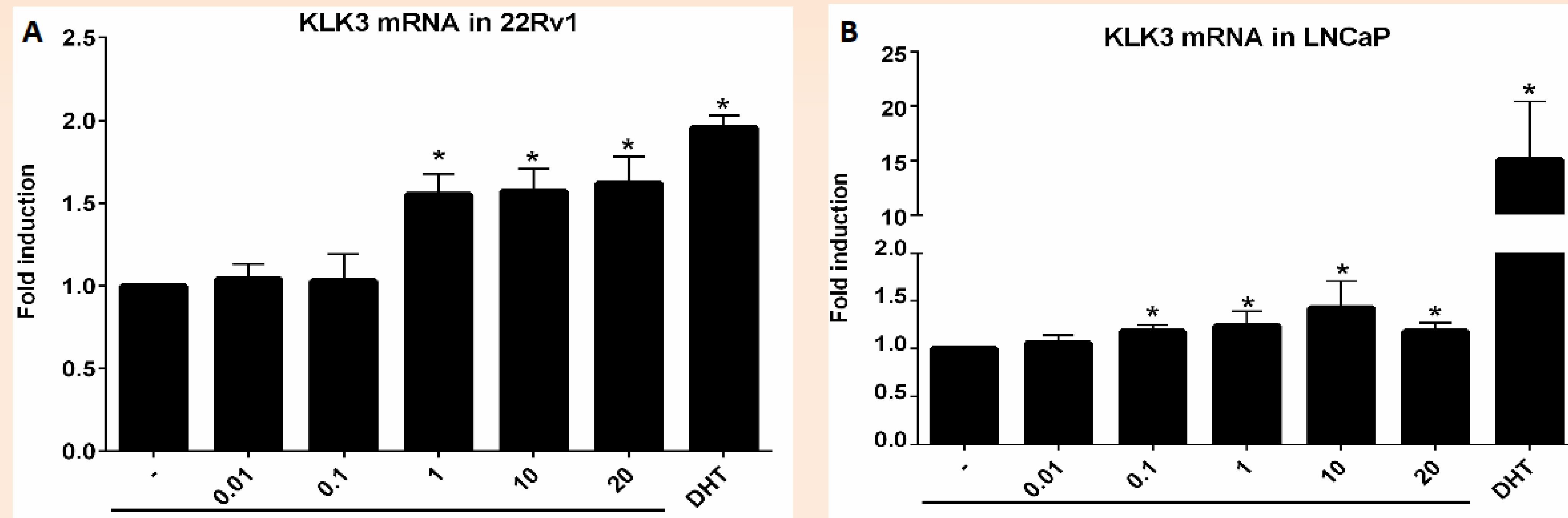

Figure 4: $22 \mathrm{Rv} 1$ and LNCap cells were incubated with different concentrations of MYC $(0,01-20 \mu \mathrm{g} / \mathrm{ml})$ or DMSO $(0,1 \% \mathrm{v} / \mathrm{v})$ as a vehicle. After $24 \mathrm{~h}$, KLK3 mRNA was determined by RT-qPCR. Results are expressed as fold induction over DMSO-treated cells \pm SD. The data are mean from 5 consecutive cell passages and were normalized per GAPDH mRNA levels. * - value is significantly different from untreated cells (DMSO; $p<0.05$ ).

MYC with presence of DHT increased induction of KLK3 mRNA more that DHT alone (almost $50 \%$ of DHT activation) in 22RV1 cells (Figure 5A). In LNCap cell line, we observed opposite effect. After 24h, MYC with DHT led to decreasing amount of KLK3 mRNA by more than 25\% (Figure 5B).
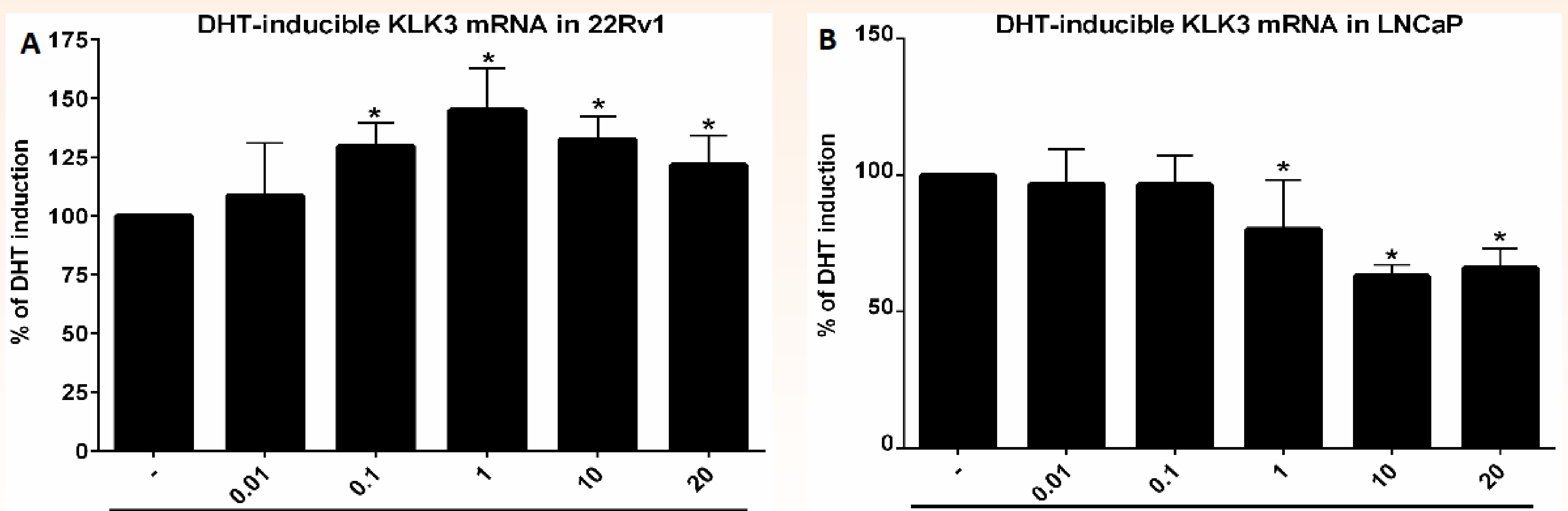

Figure 5: $22 R v 1$ and LNCap cells were incubated with different concentrations of MYC $(0,01-20 \mu \mathrm{g} / \mathrm{ml})$ and $/ 0$ DMSO $(0,1 \% \mathrm{v} / \mathrm{v})$ as a vehicle with presence of DHT (100 nM). After $24 \mathrm{~h}$, KLK3 mRNA was determined by RTqPCR. Results are expressed as $\%$ of DHT induction \pm SD. The data are mean from 5 consecutive cell passages and wer

Our previous observation of small, but significant activation of AR as well as synergistic effect of MYC on DHT-inducible KLK3 mRNA expression led us to think about possible effect of MYC on proliferation of prostate cell lines. Two lowest concentrations of MYC significantly induced proliferation of 22RV1 for $24 \mathrm{~h}$, but not for $48 \mathrm{~h}$ (Figure $6 \mathrm{~A}$ ). All other concentrations had inhibitory effect for both cell line (Figure 6A and 6B), with is probably with accordance of primary target of MYC (IMPDH inhibition).
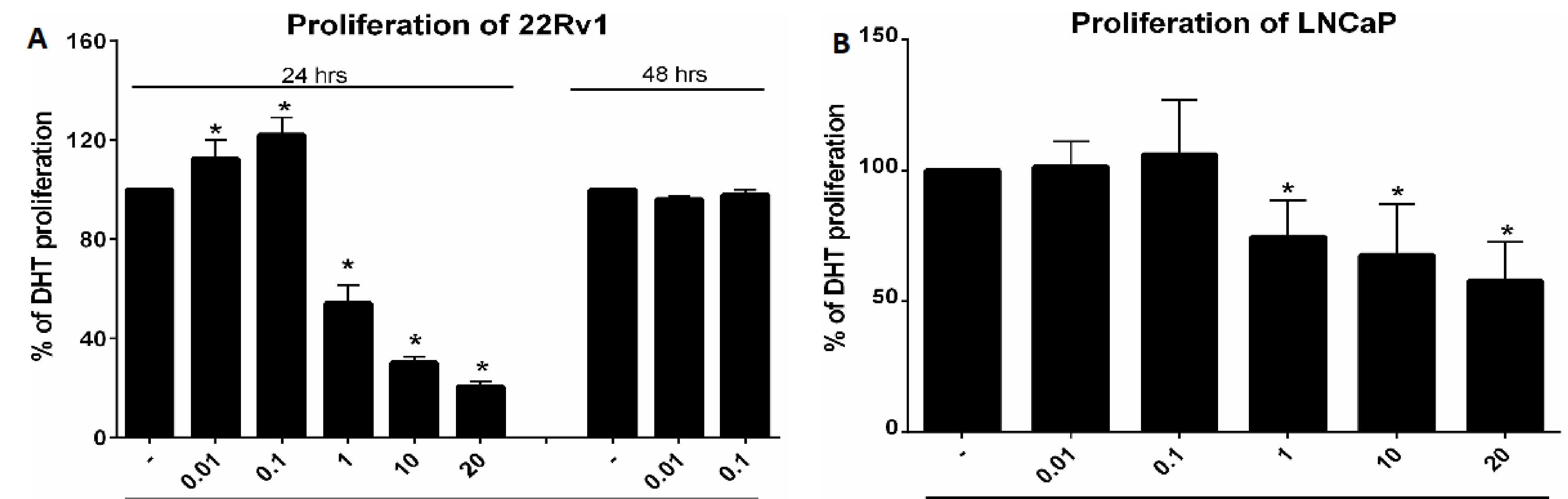

Figure 6: 22Rv1 and LNCap cells were incubated with different concentrations of MYC $(0,01-20 \mu \mathrm{g} / \mathrm{ml})$ and/or DMSO $(0,1 \% \mathrm{v} / \mathrm{v})$ as a vehicle with presence of DHT $(100 \mathrm{nM})$. After $24 \mathrm{~h}$ or $48 \mathrm{~h}$, proliferation was measured. Results are expressed as \% of DHT proliferation. ${ }^{*}$ - value is significantly different from untreated cells (DMSO; $\mathrm{p}<0.05$ ).

\section{Conclusion}

We investigated the effect of MYC on the activity of AR in two prostate cancer cell lines. We found that MYC activated androgen receptor in androgen-independent cells 22Rv1 and induced AR-target gene expression (KLK3 mRNA) in both androgen-dependent as well as independent cells. However, there was a big difference in strength of activation AR target gene KLK3 (60\% of DHT induction in $22 \mathrm{Rv} 1$ compared to $3,5 \%$ in LNCap). Moreover, the combination of MYC and DHT synergistically stimulated KLK3 mRNA induction in 22Rv1 cells while the opposite, i.e. the suppression was observed in LNCaP cells. Proliferation profile was similar for both cell lines, which can be the effect of MYC (IMPDH inhibition). This research provides novel information about the implications for widespread used transplant drug. 